\title{
Innervation of partially inserted human tendons used to reconstruct anterior cruciate ligament
}

\begin{abstract}
Aim: The human anterior cruciate ligament (ACL) provides sensory information to the nervous system that controls the activity of periarticular knee muscles. Thus, ensure the innervation of reconstructed anterior cruciate ligament lead to a better proprioception. Here we investigated the innervation of a unique case of anterior cruciate ligament reconstructed using the semitendinosus and gracilis muscle tendons maintaining the tibial insertions, 12 years after reconstruction. The study was aimed to investigate whether these pieces contain mechanoreceptors and have a pattern of innervation similar to that proper of ACL.

Materials and Methods: Immunohistochemistry for general nerve markers (neurons specific enolase, neurofilament proteins, and S100 protein) and putative mechanoproteins (acid-sensing ion channel 2 and transient receptor potential vanilloid 4) was used to map and characterize the nerves in normal ACL and the reconstructed ACL.

Results: Perivascular nerves, free nerve endings, Pacini-like and Ruffini-like corpuscles were observed, and mechanoproteins were detected in both free nerve endings and corpuscles, in normal ACL. In the ACL tendon-reconstructed ACL there was a severe reduction in the density of nerve profiles, and especially of sensory corpuscles, which were restricted to the segments closest to the tibial insertion. CONCLUSION: Normal human ACL has a rich innervation that is strongly severed after tendon-graft reconstructed ACL, even when if remains partially inserted and after long-term survival.
\end{abstract}

Keywords: anterior cruciate ligament, tendon-graft ligament reconstruction, innervation, mechanoproteins, human
Volume 3 Issue 2- 2017

\author{
Guillén I,' Guillén P,' García Suárez O,2 \\ Ordóñez $S,{ }^{3}$ Maestro A, ${ }^{4}$ Rodríguez L, ${ }^{5}$ \\ Fernández Jaen $T$,' Vega JA $\mathrm{A}^{2,6}$ \\ 'Servicio de Traumatología, Clínica CEMTRO, Spain \\ ${ }^{2}$ Departamento de Morfología y Biología Celular, Universidad de \\ Oviedo, Spain \\ ${ }^{3}$ Clínica Ordoñez, Spain \\ ${ }^{4}$ Servicios Médicos FREMAP, Spain \\ ${ }^{5}$ Servicio de Traumatología, Hospital de Cabueñes, Spain \\ ${ }^{6}$ Facultad de Ciencias de la Salud, Universidad Autónoma de \\ Chile, Chile \\ \#These authors contributed equally to this paper
}

\begin{abstract}
Correspondence: José A Vega, Departamento de Morfología y Biología Celular, Facultad de Medicina y Ciencias de la Salud Universidad de Oviedo, 33006 OVIEDO, Spain, Tel +34985104097, Fax +34985103618,Email javega@uniovi.es
\end{abstract}

Received: October 01, 2016 | Published: February 17, 2017

\section{Introduction}

Anterior cruciate ligament (ACL) ${ }^{1}$ rupture is the most common surgically treated ligament injury, and historically many efforts have been made to reconstruct ACL as anatomically as possible in order to restore knee biomechanics and prevent knee osteoarthritis., ${ }^{2,3}$ In addition to their dynamic and static mechanical roles the cruciate ligament provides sensory information to the spinal cord that regulates the activity of periarticular knee muscles. ${ }^{4-8}$ Consistently with this sensory function the knee joint capsule and ligaments, including ACL, contain several types of sensory nerve endings in which mechanotransduction takes place, i.e. mechanoreceptors. ${ }^{9,10}$ In particular, within the ACL nerve fibbers free or associate to the blood vessels, or among collagen bundles, as well as different types of sensory corpuscles (Ruffini-like, Golgi tendon organs, and Pacinianlike corpuscles) have been found. ${ }^{11-13}$ Recently, Kim \& co-workers ${ }^{14}$ have mapped the sensory innervation of human ACL. Both nerve fibbers and corpuscles were found mainly near the bony femoral attachment sites.

One interesting open question is whether or not the tendongraft reconstructed ACL reinnervates, and whether or not ACLstump remnants are important for this reinnervation. In fact, is has been suggested that maintaining the remnant of ruptured ACL the "ligamentization" of the graft accelerates contributing to a faster graft vascularization and innervation, therefore leading to a better proprioception. Nevertheless, this hypothesis is still not supported by clinical findings. ${ }^{15}$ In patients with cruciate-retaining total knee arthroplasty mechanoreceptors are present 5 to 12 years later, ${ }^{16}$ and residual remnants of ruptured ACL contain morphologically normal mechanoreceptors and proprioceptive fibbers evenly distributed at both tibial and femoral attachments in one third of the cases. ${ }^{17-20}$ Conversely, other researchers reported the absence of mechanoreceptors in biopsy samples of ACL after reconstruction with Achilles allografts (1 to 10years), and absence of sensory function after ACL re-construction, presumably because the ACL grafts were not reinnervated. ${ }^{7,21}$ To avoid nerve defects in ACL reconstruction alternative surgical procedures have been proposed. This is the case of the use of the semitendinosus and gracilis muscle tendons to reconstruct ACL maintaining their tibial insertions (Guillen, personal communication). In this way the tendon-reconstructed ACL continues to receive vascular and nerve supply from the parent muscles.

The availability of surgical pieces from long-term tendongraft reconstructed ACL is rare and few studies have analyzed the innervation of these pieces. ${ }^{14-16}$ No data have been reported using the ACL reconstruction method indicated above. Here, we used immunohistochemistry for nerve markers and mechanoproteins to analyze the long-term (12years) innervation of tendon-graft reconstructed ACL using partially disinserted semitendinosus-gracilis muscle tendons. The study was aimed to investigate whether these pieces contain mechanoreceptors and have a pattern of innervation similar to that proper of ACL. 


\section{Materials and methods}

\section{Case report}

A 44year old man was submitted to the Traumatology and Orthopedics Department, Clínica CEMTRO (Madrid, Spain) due to right knee instability. Arthroscopic exploration showed partial disruption of ACL and ligament replacement was recommended. ACL was surgically removed using arthroscopy and it was reconstructed using the tendons of the semitendinosus and gracilis muscles disinserted proximally and maintaining the tibial insertion at July 2002. Twelve years later the patient undergoes knee stability again, and was submitted to arthroscopic replacement of ACL using frozenAchilles tendon allograft.

\section{Materials}

The surgical piece removed was divided longitudinally intro two halves, fixed in $10 \%$ formaldehyde in $0.1 \mathrm{M}$ phosphate buffer saline (PBS) at $\mathrm{pH} 7.4$ for $48 \mathrm{~h}$ at $4^{\circ} \mathrm{C}$, dehydrated and embedded in paraffin. Sections $10 \mu \mathrm{m}$ were obtained, sampled each 20 sections, mounted on gelatine-coated microscope slides and processed for immunohistochemistry. One half was sectioned longitudinally, and the other one transversally. As controls, three normal ACL specimens were taken from healthy knee amputated at thigh level due to trauma (provided by Dr. A. Maestro), and processed identically as described above.

\section{Immunohistochemistry}

Indirect peroxidase-antiperoxidase immunohistochemistry was performed as follows: sections were deparaffinized and rehydrated, then rinsed in $0.05 \mathrm{M} \mathrm{HCl}$ Tris buffer $(\mathrm{pH} 7.5)$ containing $0.1 \%$ bovine serum albumin and $0.1 \%$ Triton X-100. Thereafter the endogenous peroxidase activity $(3 \% \mathrm{H} 2 \mathrm{O} 2)$ and non-specific binding were blocked with $10 \%$ foetal calf serum. The sections were incubated overnight in a humid chamber at $4^{\circ} \mathrm{C}$ with primary antibodies (Table 1). The antibodies against neuron-specific enolase (NSE) and neurofilament protein (NFP) were used as specific axon markers; antibodies against S100 protein were used to immunolabel Schwann cells and Schwannrelated cells. ${ }^{22,23}$ Antibodies against ASIC2 (acid-sensing ion channel 2) and TRPV4 (transient receptor potential vanilloid 4 ion channel) were also used to detect these two putative mechanoproteins. ${ }^{24}$ After incubation with the primary antibodies, sections were rinsed in the same buffer and incubated with Dako EnVision System labelled polymerHR anti-rabbit IgG or anti-mouse IgG (DakoCytomation, Denmark) for 30 minutes at room temperature. Finally, sections were washed and the immune reaction visualized using 3-3'-diaminobenzidine as a chromogen. To ascertain structural details, sections were slightly counterstained with hematoxylin \& eosin. To test the specificity of the immune reactivity representative sections were processed in the same way as described above using non-immune rabbit or mouse sera instead of the primary antibodies, omitting the primary antibodies in the incubation, or using pre-absorbed antibodies for ASIC2 and TRPV4 $(5 \mu \mathrm{g}$ of the blocking peptide in $1 \mathrm{ml}$ of the antibody working solution).

\section{Quantitative study}

The density of innervation in both normal and tendon-grafted ALC was performed on transversal section of each segment of ACL. In transversal sections the density of free nerve endings was quantified following the method proposed by Kim and co-workers. ${ }^{14}$ Briefly, the piece was divided into 6 segments identified as TI (tibial insertion), S2, S3, S4, S5, FI (femoral insertion), and 5 sections per segment were selected, $300 \mathrm{~mm}$ apart to avoid to measuring the same structure twice. In each section five randomly selected fields were measured $\left(2.5 \mathrm{~mm}^{2}\right)$ using an automatic image analysis system (Quantimet 550, Leika, QWIN Program) The number of nerve profiles immune reactivity for S100 protein was determined in the sub synovial layer, collagen fascicles and perivascular plexuses; moreover identified sensory corpuscles were counted. Because counts were performed in one half of the pieces the results were doubled. No statistical comparative analysis was carried out since an unique case of tendongrafted was evaluated.

Table I Primary antibodies used in the study

\begin{tabular}{|c|c|c|c|}
\hline Antigen & Origin & Dilution & Supplier \\
\hline \multicolumn{4}{|c|}{ General neural markers } \\
\hline $\begin{array}{l}\text { NSE (clone BBS/NC/ } \\
\text { VI-HI4) }\end{array}$ & Mouse & $\mathrm{I}: 1000$ & Dako' \\
\hline Pan-NF (2SII) & Mouse & 0.11111111 & Dako' \\
\hline S-100 protein & Rabbit & $\mathrm{I}: 1000$ & Dako' \\
\hline SI00P(clone 4C4.9) & Mouse & $\mathrm{I}: 1000$ & Thermo Scientific ${ }^{2}$ \\
\hline \multicolumn{4}{|l|}{ Mechanoproteins } \\
\hline ASIC2 & Rabbit & 0.1805556 & $\begin{array}{l}\text { Lifespan } \\
\text { Biosciences }^{3}\end{array}$ \\
\hline TRPV4 & Rabbit & 0.1111111 & Abcam $^{4}$ \\
\hline
\end{tabular}

ASIC2, acid-sensing ion channel 2; NSE, neuron specific enolase; TRPV4, transient receptor potential channel vanilloid 4 'Glostrup, Denmark; ${ }^{2}$ Thermo Scientific, Freemont, CA, USA; ${ }^{3}$ Seattle, WA, USA; ${ }^{4}$ Cambridge, UK

\section{Results}

\section{Normal ACL}

We first tested the occurrence of nerve bundles, isolated nerve fibres, and morphologically differentiated mechanoreceptors in samples of normal non-injured ACL. Small nerve bundles were regularly found in the vicinity of the blood vessels, in the sub synovial layer but also among the collagen fascicles (Figure 1A). They displayed immune reactivity for both axonic and Schwann-cell markers. In the same localizations isolated nerve axons (presumably ending as free nerve endings; data not shown), and several morphotypes of mechanoreceptors were found, mostly identified as simple sensory corpuscles or Pacini-like corpuscles (Figure 1B) (Figure 1C) (Figure 1E). They consisted of one to three NFP or NSE positive axons, each covered by independent continuous inner core displaying strong S100 protein immune reactivity. Outside the inner core there was a more or less wide corpuscular space and a capsule (Figure 1E). Furthermore, Ruffini-like sensory corpuscles were rarely observed (Figure 1D), as well as some other kinds of capsular corpuscles apparently multi afferented, but that surely correspond with multiple sections of an axon arranged as wool ball (Figure 1F). The density of morphologically 
defined sensory corpuscles in normal ACL is summarized in Table 2. As it can be seen the maximal densities are in the vicinity of the tibial and femoral insertions, and progressively decrease at the central segment.

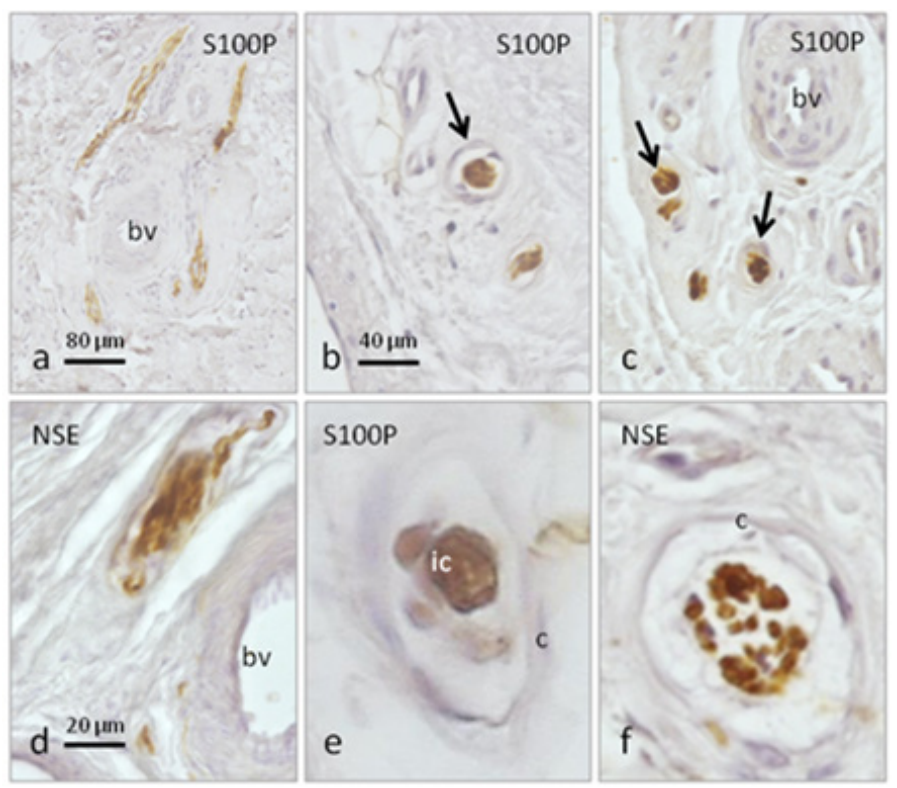

Figure I Immunohistochemical localization of general nerve markers (neuron specific enolase for axons; SI00 protein for Schwann cells and Schwann related cells in sensory corpuscles) in sections of normal anterior cruciate ligament. Perivascular nerves (A), and different kinds of sensory corpuscles were found $(B, C)$, especially Ruffini-like corpuscles (D), Pacini-like corpuscles $(E)$, and globular sensory corpuscles $(F)$.

Scale bar, $80 \mu \mathrm{m}$ for $A ; 40 \mu \mathrm{m}$ for B and C; $20 \mu \mathrm{m}$ for D,E,F.

$\mathrm{BV}$, blood vessels; $\mathrm{C}$, capsule; IC, inner core

\section{Long-term partially-disinserted grafted tendons}

Nerve profiles displaying immunoreactivity for NSE, NFP and S100 protein were observed among the collagen fascicles in the removed tendon-grafted surgical piece (Figure 2A-2C). The most noticeable difference in the pattern of innervation with respect to the normal ACL was the severe reduction in the density of nerve profiles, and especially of sensory corpuscles (Table 2). In the entire piece four structures resembling sensory corpuscles were found, and they cannot be identified as none of the canonical morphotypes types present in joints although were provided of a thick capsule. They were localized in the segments TI and P2; in all segments, however, sparse and isolated nerve fibres were also observed (Figure 2D-2G).

\section{Mechanoproteins}

The putative functional role of nerve fibres supplying the peripheral tissues, including joint tissues, can be determined on the basis of the expression of some specific proteins for mechanoreceptors, nociceptors, thermoreceptors, and so. Nerve fibres in ACL can be regarded a priori as nociceptive or mechano-proprioceptive in nature. To investigate whether or not they work as mechanoceptive we have investigated the expression of two putative mechanoproteins within them, i.e. ASIC2 and TRPV4. In normal ACL Ruffini-like corpuscles were found to display ASIC2 and TRPV4 immunoreactivity (Figure 3A \& Figure 3C), as well as the capsulated multiafferented corpuscles (Figure 3B) and the free nerve fibres (Figure 3D). In the tendon-grafted surgical piece ASIC2 and TRPV immunoreactivity were detected in small nerve bundles (Figure 3E \& Figure 3F) and perivascular free nerve fibres (Figure $3 \mathrm{G}$ ), but never in structures resembling sensory corpuscles. The density of mechanoceptive nerve fibres was reduced in these pieces compared with normal ACL.

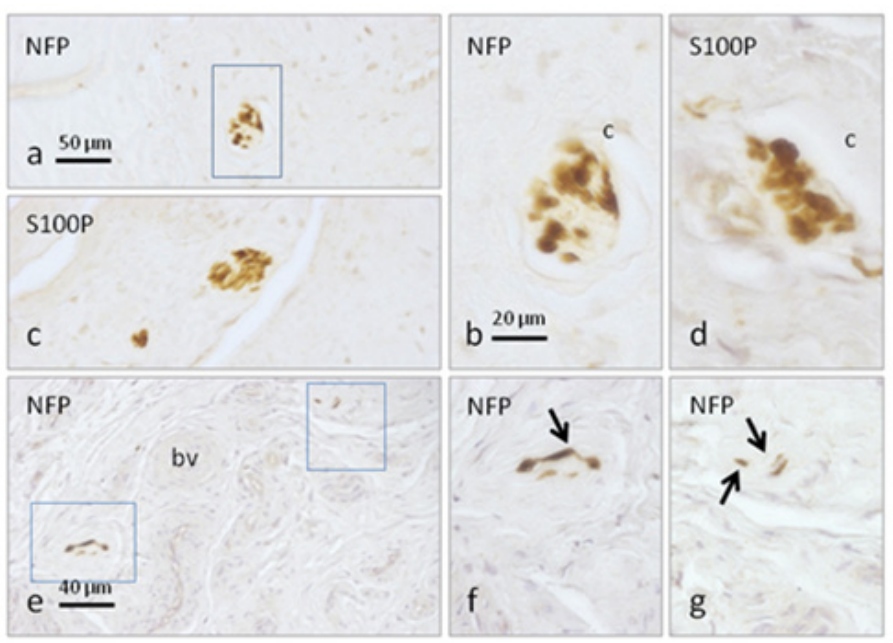

Figure 2 Immunohistochemical localization of general nerve markers (neurofilaments for axons; SIO0 protein for Schwann cells and Schwann related cells in sensory corpuscles) in sections of semitendinosus and gracilis tendon muscles used to reconstruct anterior cruciate ligament 12years ago. Scarce capsulated sensory corpuscles (A-D) and isolated nerve fibbers (E-G) were observed. Scale bar, $50 \mu \mathrm{m}$ for $A$ and C; $40 \mu \mathrm{m}$ for $\mathrm{E} ; 20 \mu \mathrm{m}$ for $\mathrm{B}, \mathrm{D}, \mathrm{F}$ and $\mathrm{G}$, arrows indicate nerve fibbers. $\mathrm{BV}$, blood vessels; C, capsule

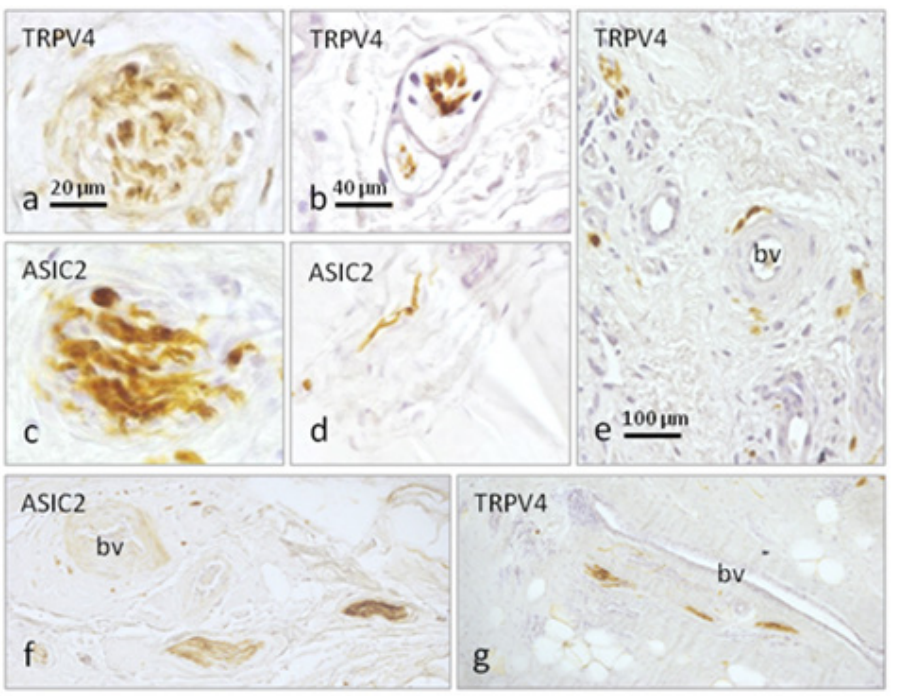

Figure 3 Immunohistochemical localization of the putative mechanoproteins ASIC2 and TRPV4 in sections of normal ACL (A-D) and in sections of semitendinosus and gracilis tendon muscles used to reconstruct anterior cruciate ligament 12 years ago (E-F). Ruffini-like corpuscles display ASIC2 and TRPV4 immunoreactivity in normal ACL $(A, C)$, as well as the capsulated multiafferented corpuscles (B) and the free nerve fibres (D). In the tendon-grafted surgical piece ASIC2 and TRPV immunoreactivity were detected in small nerve bundles $(E, F)$ and perivascular free nerve fibres (9). Scale bar, $20 \mu \mathrm{m}$ for A and C; $40 \mu \mathrm{m}$ for B and D; $100 \mu \mathrm{m}$ E,F,G. $\mathrm{BV}$, blood vessels. 
Table 2 Density of sensory corpuscles and free nerve ending in the normal anterior cruciate ligament and in ACL reconstructed using the tendons of the semitendinosus and gracilis muscles maintaining the tibial insertion

\begin{tabular}{llllll}
\hline (\% area) & & $\begin{array}{l}\text { NB, FNE } \\
\text { and PVN }\end{array}$ & P-LC & R-L C & NTC \\
\hline Control ACL & T & $3.1 \pm 1.1$ & 3.2 & 2.3 & \\
& S2 & $2.3 \pm 0.3$ & 2.2 & 1.3 & \\
& S3 & $2.2 \pm 0.4$ & & 1.1 & \\
& S4 & $1.2 \pm 0.2$ & & 1.2 & \\
& S5 & $2.8 \pm 0.9$ & 2.4 & 2.2 & \\
Tendon-grafted & & & & & \\
ACL & T & $3.1 \pm 1.6$ & 4.3 & 1 & \\
& $2.1 \pm 0.3$ & & & 2 & \\
& S3 & $2.1 \pm 0.6$ & & & 2 \\
S2 & S4 & $2.3 \pm 1.1$ & & & I \\
& S5 & $1.2 \pm 0.5$ & & & I \\
& F & $0.6 \pm 0.1$ & & & 0 \\
\hline
\end{tabular}

F, femoral; FNE, free nerve endings; NB, nerve bundles; NTC, non-typica corpuscles; P-LC, pacinian corpuscles; R-LC, ruffini-like corpuscles; SSL, subsynovial layer; Others, other kinds of sensory corpuscles; PVN, perivascular nerves

\section{Discussion}

Surgical ACL reconstruction using tendon grafts has become the standard treatment for functionally unstable ACL-deficient knee. Although tendons clearly differ biologically from ligaments, multiple experimental studies have shown that the implanted tendons indeed seem to remodel into a ligamentous "anterior cruciate ligament-like" structure, in a biological process known as "ligamentization". ${ }^{25,26}$ This study was designed to analyze the innervation of the tendons of the semitendinosus and gracilis muscles partially disinserted used to reconstruct ACL. It was a single case performed 12 years before. The surgical procedure included disinsertion of these tendons only proximally whereas the distal (tibial) insertion was maintained, therefore retaining blood vessels and nerves supply.

It is well know that ACL provides information to the central nervous system to control dynamic and static activity of the periarticular knee muscles. ${ }^{8}$ Thus to preserve or to ensure the innervation of the reconstructed ACL should be a main scope of ACR reconstructive surgery. ${ }^{4-6}$ Sensory innervation is absent from reconstructed $\mathrm{ACL}^{7,21}$ while innervation has been demonstrated in grafts inserted in ACL remnants. ${ }^{17-20}$

The pattern of innervation of the normal ACL we have observed here basically agrees with previous studies using gold impregnation or immunohistochemical techniques, ${ }^{11,12,14}$ and also was quite similar to that reported in ACL remnants. ${ }^{17,20}$ Perivascular nerves, free nerve endings and two types of identifiable mechanoreceptors, i.e. Pacinilike and Ruffini-like corpuscles, were regularly observed. In our hands no other kinds of sensory corpuscles were found. On the other hand, the density of innervation varied along LCA, being denser in the segments near the femoral and tibial insertions, and minimal in the central segments, especially the occurrence of mechanoreceptors.
Regarding the innervation of the reconstructed ACL we have found that the density of innervation was markedly reduced, especially at the proximal (femoral) stump were scarce nerve fibbers and no sensory corpuscles were found. Free nerve endings and atypical morphotypes of sensory corpuscles were found; they were capsulated and resembled Ruffini-like corpuscles; in no case proper Golgi tendon organs were observed. ${ }^{27}$ Thus, ACL reconstructed using partially inserted tendons retain innervation even for long periods of time, but it was restricted to the segments close to the distal (tibial) insertion. Presumably the nervous apparatus of these LCA come from the tibial extreme of the tendon, more probably that from the femoral bone-anchored stump. On the other hand, whether the sensory corpuscle we have found are surviving of the original tendons or are new-formed after graft cannot be known. In other territories, like skin, denervated sensory corpuscles degenerated ${ }^{28}$ but whether or not this also occurs in tendons has not been investigated. Interestingly, abundant nerve profiles were found in the sub synovial tissue of normal ACL while they were scarce in the surface, no covered by typical synovial, of the grafted tendons. All together these data suggest that long-term tendon-graft reconstructed ACL are severely denervated.

To determine what modality of sensitivity is affected, nociceptive or proprioceptive, we have investigated the occurrence of two putative mechanoproteins, ASIC2 and TRPV4, in the ACL nerves. Previous studies in humans and monkeys have demonstrated the occurrence of these proteins in cutaneous mechanoreceptors and mechanosensory neurons. ${ }^{29,30}$ In recent years evidence has emerged that at the basis of the sensory processes is the activation of ion channels for the conversion of a stimulus into an electrical signal. Thus, different ion channels are currently being considered as candidates for nociception or mechanoception..$^{24,31}$ The present results provide new data about the occurrence of putative mechanoproteins in sensory corpuscles, and are the first evidence of the presence of those proteins in the nerves of LCA and tendon-reconstructed LCA. Recently several mechanoproteins were detected in human periodontal ligament, associated and non-associated to neural elements, in particular Ruffinilike corpuscles. ${ }^{32-34}$ All together, the present results demonstrate that normal human ACL has a rich innervation that is strongly severed after tendon-graft reconstructed ACL, even when if remains partially inserted and after long-term survival. A main component of the lost nervous apparatus can be regarded as mechanoceptive on the basis or its immunohistochemical profile. However, because ASIC2 and TRPV4 are polymodal channels that responds to nociception in addition to mechanical forces, ${ }^{35,36}$ an impairment of nociception cannot be ruled out. Further studies are necessaries to elucidate the role of putative mechanoproteins in human ligaments, including ACL.

\section{Acknowledgements}

None.

\section{Conflict of interest}

Author declares that there is no conflict of interest.

\section{References}

1. Duthon VB, Barea C, Abrassart S, et al. Anatomy of the anterior cruciate ligament. Knee Surg Sports Traumatol Arthrosc. 2006;14(3):204-213.

2. Muller B, Duerr ER, van Dijk CN, et al. Anatomic anterior cruciate ligament reconstruction: reducing anterior tibial subluxation. Knee Surg Sports Traumatol Arthrosc. 2016;24(9):3005-3010. 
3. Rodriguez Merchan EC. Evidence-based ACL reconstruction. Arch Bone Jt Surg. 2015;3(1):9-12.

4. Johansson H, Sjölander P, Sojka P. Receptors in the knee joint ligaments and their role in the biomechanics of the joint. Crit Rev Biomed Eng. 1991;18(5):341-368.

5. Johansson H, Sjölander P, Sojka P. A sensory role for the cruciate ligaments. Clin Orthop Relat Res. 1991;268:161-178.

6. Solomonow M, Krogsgaard M. Sensorimotor control of knee stability. A review. Scand J Med Sci Sports. 2001;11(2):64-80.

7. Krogsgaard MR, Fischer Rasmussen T, Dyhre Poulsen P. Absence of sensory function in the reconstructed anterior cruciate ligament. $J$ Electromyogr Kinesiol. 2011;21(1):82-86.

8. Ward S, Pearce AJ, Pietrosimone B, et al. Neuromuscular deficits after peripheral joint injury: a neurophysiological hypothesis. Muscle Nerve. 2015;51(3):327-332.

9. Zimny ML. Mechanoreceptors in articular tissues. Am $J$ Anat. 1988;182(1):16-32.

10. Del Valle ME, Harwin SF, Maestro A, et al. Immunohistochemical analysis of mechanoreceptors in the human posterior cruciate ligament: a demonstration of its proprioceptive role and clinical relevance. $J$ Arthroplasty. 1998;13(8):916-922.

11. Zimny ML, Schutte M, Dabezies E. Mechanoreceptors in the human anterior cruciate ligament. Anat Rec. 1986;214(2):204-209.

12. Schutte MJ, Dabezies EJ, Zimny ML, et al. Neural anatomy of the human anterior cruciate ligament. J Bone Joint Surg Am. 1987;69(2):243-247.

13. Krauspe R, Schmitz F, Zöller G, et al. Distribution of neurofilamentpositive nerve fibbers and sensory endings in the human anterior cruciate ligament. Arch Orthop Trauma Surg. 1995;114(4):194-198.

14. Kim KM, Chun $\mathrm{CH}$, Kim BG. The histologic mapping of sensory innervation in the human anterior cruciate ligament. Korean J Sport Med. 2012;30(2):130-136.

15. Papalia R, Franceschi F, Vasta S, et al. Sparing the anterior cruciate ligament remnant: is it worth the hassle? Br Med Bull. 2012;104:91-111.

16. Zhang K, Mihalko WM. Posterior cruciate mechanoreceptors in osteoarthritic and cruciate-retaining TKA retrievals: a pilot study. Clin Orthop Relat Res. 2012;470(7):1855-1859.

17. Georgoulis AD, Pappa L, Moebius U, et al. The presence of proprioceptive mechanoreceptors in the remnants of the ruptured ACL as a possible source of re-innervation of the ACL autograft. Knee Surg Sports Traumatol Arthrosc. 2001;9(6):364-368.

18. Dhillon MS, Bali K, Vasistha RK. Immunohistological evaluation of proprioceptive potential of the residual stump of injured anterior cruciate ligaments (ACL). Int Orthop. 2010;34(5):737-741.

19. Lee BI, Min KD, Choi HS, et al. Immunohistochemical study of mechanoreceptors in the tibial remnant of the ruptured anterior cruciate ligament in human knees. Knee Surg Sports Traumatol Arthrosc. 2009;17(9):1095-1101.

20. Bali K, Dhillon MS, Vasistha RK, et al. Efficacy of immunohistologica methods in detecting functionally viable mechanoreceptors in the remnant stumps of injured anterior cruciate ligaments and its clinical importance. Knee Surg Sports Traumatol Arthrosc. 2012;20(1):75-80.
21. Kim $\mathrm{SH}$, Chun $\mathrm{CH}$, Chun $\mathrm{KC}$, et al. Histological assessment of mechanoreceptors in Achilles allografts after anterior cruciate ligament reconstruction. Am J Sports Med. 2012;40(9):2061-2065.

22. Vega JA, García Suárez O, Montaño JA, et al. The Meissner and Pacinian sensory corpuscles revisited new data from the last decade. Microsc Res Tech. 2009;72(4):299-309.

23. Vega JA, Haro JJ, Del Valle ME. Immunohistochemistry of human cutaneous Meissner and pacinian corpuscles. Microsc Res Tech. 1996;34(4):351-361

24. Del Valle ME, Cobo T, Cobo JL, et al. Mechanosensory neurons, cutaneous mechanoreceptors, and putative mechanoproteins. Microsc Res Tech. 2012;75(8):1033-1043.

25. Claes S, Verdonk P, Forsyth R, et al. The "ligamentization" process in anterior cruciate ligament reconstruction: what happens to the human graft? A systematic review of the literature. Am J Sports Med. 2011;39(11):2476-2483.

26. Pauzenberger L, Syré S, Schurz M. "Ligamentization" in hamstring tendon grafts after anterior cruciate ligament reconstruction: a systematic review of the literature and a glimpse into the future. Arthroscopy. 2013;29(10):1712-1721.

27. Banks RW, Hulliger M, Saed HH, et al. A compative analysis of the encapsulated end-organs of mammalian skeletal muscles and their sensory nerve endings. J Anat. 2009;214(6):859-887.

28. Zelena J. Nerves and mechanoreceptors. London: Ed Chapman \& Hall; 1994. 356p.

29. Calavia MG, Montaño JA, García Suárez O, et al. Differential localization of Acid-sensing ion channels 1 and 2 in human cutaneuspacinian corpuscles. Cell Mol Neurobiol. 2010;30(6):841-848.

30. Cabo R, Alonso P, Viña E, etal.ASIC2 is present in human mechanosensory neurons of the dorsal root ganglia and in mechanoreceptors of the glabrous skin. Histochem Cell Biol. 2015;143(3):267-276.

31. Belmonte C, Viana F. Molecular and cellular limits to somatosensory specificity. Mol Pain. 2008;4:14.

32. Rahman F, Harada F, Saito I, et al. Detection of acid-sensing ion channel 3 (ASIC3) in periodontal Ruffini endings of mouse incisors. Neurosci Lett. 2011;448(2):173-177.

33. Fernández González FJ, Cañigral A, López Caballo J, et al. Recombinant osteoprotegerin effects during orthodontic movement in a rat model. Eur J Orthod. 2015;38(4):379-385

34. Son GY, Yang YM, Park WS, et al. Hypotonic stress induces RANKL via transient receptor potential melastatin 3 (TRPM3) and vaniloid 4 (TRPV4) in human PDL cells. J Dent Res. 2015;94(3):473-481.

35. Nilius B, Owsianik G. The transient receptor potential family of ion channels. Genome Biol. 2011;12(3):218.

36. Sherwood TW, Frey EN, Askwith CC. Structure and activity of the acid sensing ion channels. Am J Physiol Cell Physiol. 2012;303(7):C699C710. 